\title{
Article \\ Experimental Analysis of Plantar Fascia Mechanical Properties in Subjects with Foot Pathologies
}

\author{
Silvia Todros ${ }^{1}\left(\mathbb{D}\right.$, Carlo Biz $^{2, *(1)}$, Pietro Ruggieri ${ }^{2}(\mathbb{C})$ and Piero G. Pavan ${ }^{1}$ \\ 1 Department of Industrial Engineering, University of Padova, 35131 Padova, Italy; \\ silvia.todros@unipd.it (S.T.); piero.pavan@unipd.it (P.G.P.) \\ 2 Orthopaedic and Traumatology Clinic, Department of Surgery, Oncology and Gastroenterology DiSCOG, \\ University of Padova, 35128 Padova, Italy; pietro.ruggieri@unipd.it \\ * Correspondence: carlo.biz@unipd.it; Tel.: +39-049-821-3239
}

Citation: Todros, S.; Biz, C.; Ruggieri, P.; Pavan, P.G. Experimental Analysis of Plantar Fascia Mechanical Properties in Subjects with Foot Pathologies. Appl. Sci. 2021, 11, 1517. https://doi.org/10.3390/ app11041517

Academic Editor: Arkady Voloshin Received: 12 January 2021

Accepted: 5 February 2021

Published: 8 February 202

Publisher's Note: MDPI stays neutral with regard to jurisdictional claims in published maps and institutional affiliations.

Copyright: (c) 2021 by the authors. Licensee MDPI, Basel, Switzerland. This article is an open access article distributed under the terms and conditions of the Creative Commons Attribution (CC BY) license (https:// creativecommons.org/licenses/by/ $4.0 /)$.

\begin{abstract}
Plantar Fascia (PF) is a fibrous tissue that plays a key role in supporting the foot arch; it can be affected by several pathologies that can alter foot biomechanics. The present study aims at investigating the mechanical behavior of PF and evaluating possible correlations between mechanical properties and specific pathologies, namely diabetes and plantar fibromatosis (Ledderhose syndrome). PF samples were obtained from 14 human subjects, including patients with Ledderhose syndrome, patients affected by diabetes and healthy subjects. Mechanical properties of PF tissues were evaluated on three samples from each subject, by cyclic uniaxial tensile tests up to $10 \%$ of maximum strain and stress relaxation tests for $300 \mathrm{~s}$, in hydrated conditions at room temperature. In tensile tests, PF exhibits non-linear stress-strain behavior, with a higher elastic modulus (up to 25-30 MPa) in patients affected by Ledderhose syndrome and diabetes with respect to healthy subjects (elastic modulus $10 \div 14 \mathrm{MPa}$ ). Stress-relaxation tests show that PF of patients affected by Ledderhose syndrome and diabetes develop more intense viscous phenomena. The results presented in this work represent the first experimental data on the tensile mechanical propertied of PF in subjects with foot diseases and can provide an insight on foot biomechanics in pathological conditions.
\end{abstract}

Keywords: plantar fascia; plantar fibromatosis; diabetes; mechanical testing; viscoelasticity

\section{Introduction}

The Plantar Fascia (PF) is a connective tissue composed of type I collagen fibers arranged in a longitudinal direction, elastic fibers and an extracellular matrix highly rich in hyaluronan [1]. This thin fibrous band connects the plantar heel to the metatarsophalangeal joints [2]. PF is thought to be attached to the Achilles tendon via the superficial aligned trabeculae of the calcaneus, even if the continuity of collagen fibers between the Achilles tendon and the PF through the calcaneus is still debated [3]. PF has a fundamental biomechanical role in supporting the medial longitudinal arch of the foot [4-6] and is capable of storing strain energy and converting it into propulsive force, behaving as a quasi-elastic tissue [7], but it is in general characterized by relevant viscoelastic behavior [8]. Moreover, it has been recently demonstrated that fascia play an important role in proprioception, compartment syndromes and fibromyalgia [2,5]. The most common pathological condition that the PF can undergo is plantar fasciitis [9], which typically affects workers and athletes whose activities have a high impact on the foot, causing inferior heel pain [10,11]. Another pathology affecting the PF is plantar fibromatosis, also called Ledderhose disease, which is a benign nodular formation usually in the medial part of the PF causing pain during gait $[12,13]$. Such disease is commonly treated by a partial or total aponeurotomy, although other options have recently been described [14,15]. In addition to diseases directly involving the PF, there are various systemic pathologies, primarily diabetes, that affect foot biomechanics in different ways. Diabetic patients are subject to foot problems for multifactorial reasons [16]: Peripheral neuropathy, peripheral vascular disease, delayed bone 
healing and altered immune function resulting in a diabetic foot with chronic ulcers and neuropathic arthropathy that overall subverts the normal biomechanics of the foot $[17,18]$. Recent studies have focused on the importance of understanding the biomechanics of the diabetic foot to define the appropriate treatment and prevent complications for each of its different pathological aspects $[19,20]$. This investigation must involve also the PF, as one of the elements of the foot region assuming distinctive structural behavior. For example, a selective PF release has been suggested to redistribute the pressure on the sole for healing forefoot chronic ulcers in diabetic patients [21].

Few works in the literature have been carried out on the experimental testing of the mechanical properties of PF [3,8,22]; moreover, the possible effects of structural impairments on the mechanical behavior of fascia are not well understood.

Experimental mechanical studies on PF of patients affected by Ledderhose disease are almost absent, even though clinical evidence show that the healthy fascial tissue is progressively replaced by abnormal thick collagen fibers, leading to sclerosis of the entire PF [23], associated with an overall foot stiffening effect. This is generally complemented by an inflammatory reaction of the tissues around the nodules, causing pain and swelling that can lead to impairments in foot biomechanics [24].

Some studies have evaluated the PF properties in the diabetic foot. It is known that diabetes causes an increased thickness of Achilles tendon and PF, more evident in the presence of neuropathy, and it is supposed that this could contribute to an increase in the tensile force maintained throughout the whole gait cycle. The thickness of the PF and the Achilles tendon was measured by means of ultrasound tests highlighting a thickening of both these structures in patients affected by diabetes, in particular in those who were affected also by neuropathy [21]. The thickening of the PF was associated by the authors with a more rigid foot, leading to alterations of the gait cycle and of the plantar pressure distribution. Fabrikant et al. [25] performed ultrasound tests on patients affected by plantar fasciitis, highlighting a thickening of the PF due to this disease, while a decrease in thickness was observed after clinical treatments, suggesting that the PF thickening could be related to inflammation episodes.

While the PF thickness is considered as a parameter which shows a correlation with pathological conditions, other studies remark its strong variability depending on the PF region, including medial, lateral and central components, and on other factors, such as sex or body mass index (BMI) [26,27].

The present study has the purpose of investigating if the Ledderhose disease and diabetes could be associated with differences in the PF mechanical response, with focus on the nonlinear stress-strain behavior and viscoelastic properties. Coupling mechanical tests to other clinical observations could provide information on tissue damages and additional diagnostic parameters regarding foot pathologies.

\section{Materials and Methods}

\subsection{Subjects}

The PF of 14 subjects (Table 1) were excised. All subjects participating in this study received a thorough explanation of the risks and benefits of inclusion and gave their oral and written informed consent to publish the data. The study was performed in accordance with the ethical standards of the 1964 Declaration of Helsinki as revised in 2013 [28] and approved by the Ethics Committee of the University of Padova and the National Institutes of Health (protocol code 3513/AO/15; date of approval: 28 January, 2016). 
Table 1. Characteristics of the subjects involved in the study.

\begin{tabular}{|c|c|c|c|c|c|c|c|c|c|c|c|c|c|c|}
\hline & \multicolumn{9}{|c|}{ Pathologic } & \multicolumn{5}{|c|}{ Healthy } \\
\hline & \multicolumn{5}{|c|}{ Ledderhose Disease } & \multicolumn{4}{|c|}{ Diabetes } & & & & & \\
\hline subject ID code & L1 & $\mathrm{L} 2$ & L3 & $\mathrm{L} 4$ & L5 & D1 & D2 & D3 & D4 & $\mathrm{H} 1$ & $\mathrm{H} 2$ & H3 & $\mathrm{H} 4$ & H5 \\
\hline sex & M & $\mathrm{F}$ & $\mathrm{F}$ & M & $\mathrm{F}$ & M & $\mathrm{F}$ & M & $\mathrm{M}$ & $\mathrm{F}$ & $\mathrm{M}$ & $\mathrm{M}$ & M & $\mathrm{F}$ \\
\hline mass $(\mathrm{kg})$ & 80 & 88 & 55 & 60 & 72 & 70 & 55 & 78 & 92 & 50 & 82 & 136 & 75 & 68 \\
\hline height (cm) & 175 & 156 & 160 & 175 & 170 & 165 & 160 & 180 & 170 & 155 & 178 & 183 & 165 & 185 \\
\hline $\operatorname{BMI}\left(\mathrm{kg} / \mathrm{m}^{2}\right)$ & 26.1 & 36.2 & 21.5 & 19.6 & 24.9 & 25.7 & 21.5 & 24.1 & 31.8 & 20.8 & 25.9 & 40.6 & 27.5 & 19.9 \\
\hline age $(y)$ & 50 & 50 & 42 & 32 & 60 & 76 & 65 & 72 & 65 & 82 & 78 & 67 & 67 & 81 \\
\hline
\end{tabular}

In five subjects, labelled with codes L1 to L5, the PF was removed due to the Ledderhose disease. Four subjects with clinical history of diabetic foot, labelled with codes D1 to D4, underwent above-knee amputation due to vascular complications. One patient (H1) underwent above-knee amputation due to complete dislocation of the knee arthroplasty and refusal to be treated with other options. This patient was affected by hypertension, but can be considered a healthy control, since this syndrome does not affect the mechanical properties of PF. Other PF was dissected from four cadaveric subjects (H2 to H5) without any foot pathologies.

\subsection{Dissection and Treatment of Samples}

None of the feet of the involved subjects had signs of infection or had previously been treated surgically. Excised feet were dissected through a longitudinal cutaneous incision along the midline from the heel to the third toe. First, the skin was cut and raised medially and laterally, then the subcutaneous tissue was carefully removed to expose the PF. The fascia was isolated proximally to its calcaneal origin and distally up to the insertion in the metatarsophalangeal joints, removing muscle insertions and connections with contiguous structures. PF was treated with caution to prevent any tissue damage during dissection. In case of Ledderhose disease, nodular formation and immediately adjacent PF tissue were removed.

After dissection, the PF were kept at $-20^{\circ} \mathrm{C}$ and unfrozen approximately one hour before mechanical testing by immersing them in physiological solution (PBS, phosphate buffered solution) at room temperature.

PF was cut along the proximal-distal direction to obtain three rectangular samples for each subject, with a total sample length of $25 \mathrm{~mm}$ and nominal width of $5 \mathrm{~mm}$. This number of samples was selected to obtain well balanced data for all the subjects, based on the area of the smallest available PF among different subjects.

A portion of PF dissected from a patient and the typical cut of samples for mechanical testing are shown in Figure 1a. The effective width and thickness of each sample were measured on images taken from top and side views of the sample positioned on a flat surface. The images were analyzed with the software ImageJ (NIH) [29].
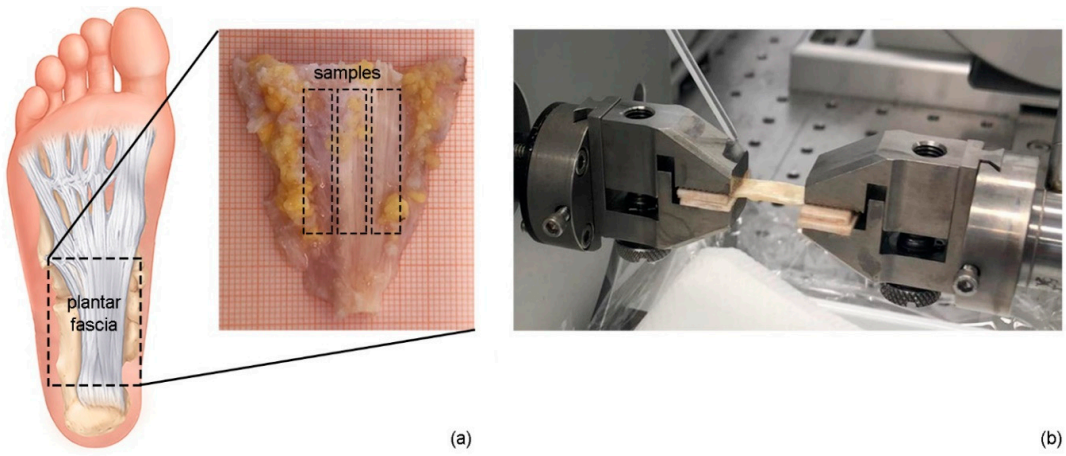

Figure 1. Portion of dissected Plantar Fascia (PF) from midfoot region and disposition of samples cut along the fiber direction (a); lateral view of gripped PF sample under mechanical testing (b). 


\subsection{Mechanical Testing}

Each end of the sample was glued between two patches of balsa wood to prevent any slippage $[30,31]$, clamped by the grips and then hydrated by dropping the PBS on the sample surface for the entire duration of the test (Figure 1b). A gauge length of $15 \mathrm{~mm}$ was selected to ensure a mean aspect ratio (length/width) of about 3, in accordance with other test protocols $[8,32]$. The samples were stiff enough to be placed flat between grips with almost null force acting $(<0.01 \mathrm{~N})$. Mechanical tests were carried out by means of Bose ElectroForce ${ }^{\circledR}$ Planar Biaxial Test Bench instrument (TA Instruments, New Castle, DE, USA) under displacement control with precision of $\pm 0.001 \mathrm{~mm}$ and adopting a load cell of $22 \mathrm{~N}$ with a precision of $\pm 0.02 \mathrm{~N}$. All the tests were carried out at room temperature $\left(25 \pm 1{ }^{\circ} \mathrm{C}\right)$. Uniaxial tensile tests consisted of five loading-unloading cycles at a constant strain rate of $10 \% \mathrm{~s}^{-1}$ by varying motor displacement up to a maximum nominal strain of $4 \%, 6 \%, 8 \%$ and $10 \%$, separated by a rest period of $60 \mathrm{~s}$. Stress-relaxation tests were carried out by elongating the sample at a fast strain rate of $100 \% \mathrm{~s}^{-1}$ and maintaining a constant strain for $300 \mathrm{~s}$ at each different level of applied strain $(4 \%, 6 \%, 8 \%$ and $10 \%)$. The samples were then brought back to initial length and kept in this condition for $300 \mathrm{~s}$. Nominal strain $\varepsilon$ was evaluated by measuring the displacement of the grips and dividing it by the initial gauge length of the sample. The nominal stress $P$ was calculated as the ratio between the force, measured by the load cell, and the initial transversal area of the sample.

The loading protocol of mechanical tests in terms of nominal strain vs. time and typical response of a PF sample in terms of nominal stress $P$ vs. time are shown in Figure 2. For each stress-relaxation test, normalized stress $P_{\text {norm }}$ was calculated as the ratio of stress at the current time $t$ and the maximum stress measured at the initial time of the relaxation process. Mean values of nominal stress $P$ vs. $\varepsilon$ in tensile tests and mean values of normalized stress $P_{\text {norm }}$ vs. $t$ in stress-relaxation tests were calculated for each subject, with associated standard error of the mean (SE). The secant elastic modulus Es was calculated from the mean of tensile data of each subject, as the slope of the straight line drawn from the origin of the stress-strain diagram and intersecting the mean curve at $8 \%$ strain.
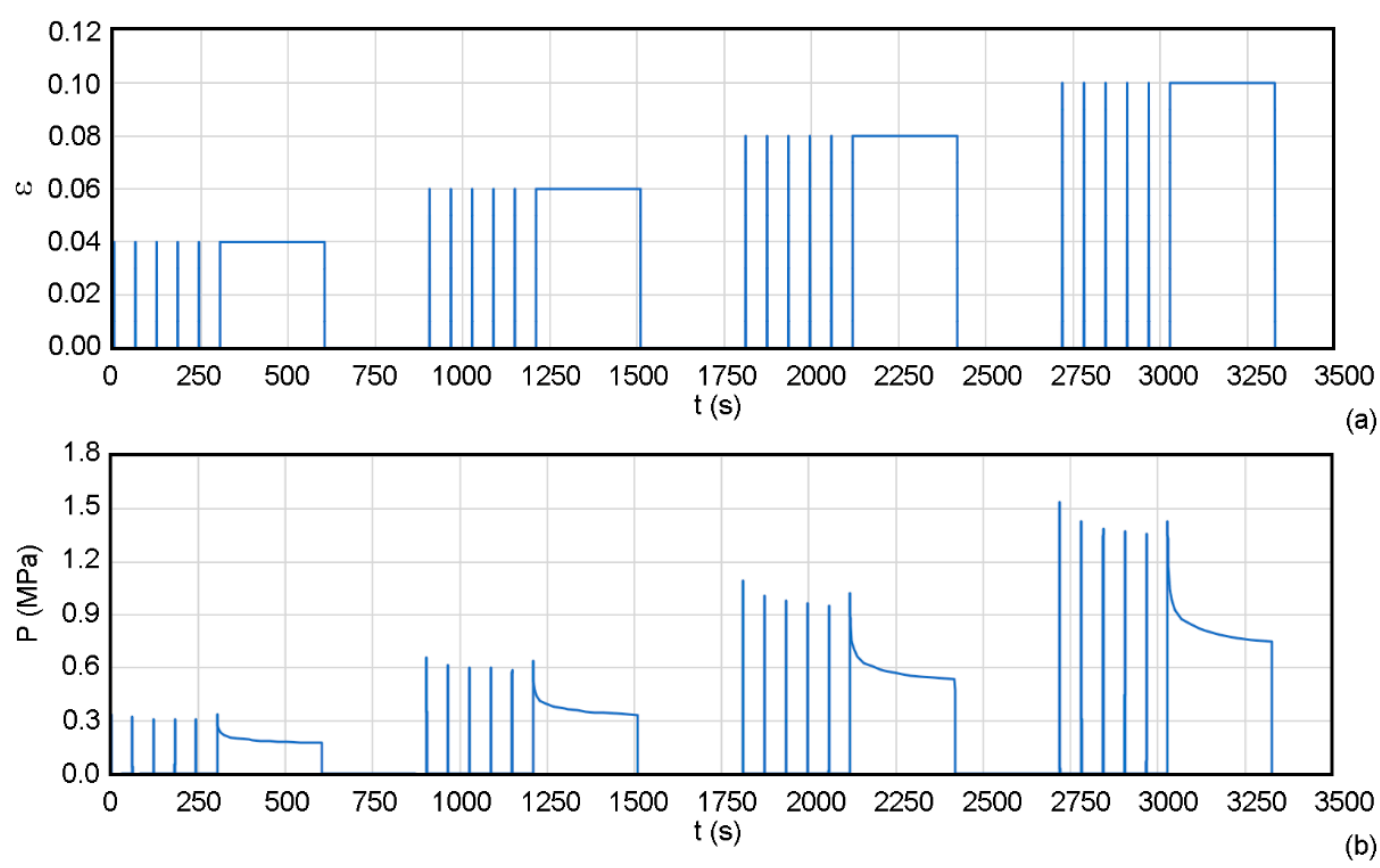

Figure 2. Loading protocol of mechanical tests in terms of nominal strain $\varepsilon$ vs. time (a) and typical response of a PF sample in terms of nominal stress P vs. time (b). 


\subsection{Statistical Analysis}

Due to the limited number of samples at disposal the Analysis of Variance (ANOVA) was based on the Kruskal-Wallis test, assuming a $p$-value of 0.05 as a maximum value to have a statistically significant difference. The possible dependency between some variables was investigated through the Pearson correlation. The statistical analysis was performed with MATLAB (Matworks ${ }^{\circledR}$ ).

\section{Results}

The typical stress-strain response of a PF sample during five loading-unloading cycles is shown in Figure 3, at 10\% strain. The comparison of consecutive cycles highlights a decrease of the stress values with the increase in the number of cycles, until stabilization. From the 1st to the 2 nd cycle, a decrease of $12 \%$ of the maximum stress is found. The percentage reduction progressively diminishes below $3 \%$ from the 4 th to the 5 th cycle; after that, the mechanical response is considered stable.

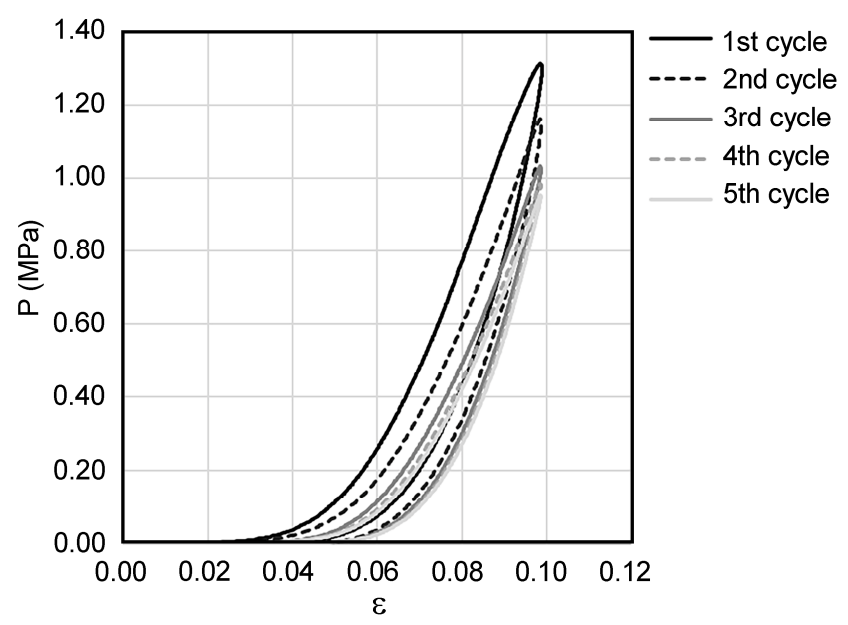

Figure 3. Mechanical response of a PF sample, in terms of nominal stress P vs. nominal strain $\varepsilon$, during five loading-unloading cycles at $10 \%$ strain.

The experimental results of the 5th cycle of tensile tests at $10 \%$ strain are considered to calculate the mean values of nominal stress $P$ for each subject (Figure 4), comparing Ledderhose disease, diabetes and healthy subjects. PF shows nonlinear stress-strain behavior with increasing stiffness at higher strain levels in both healthy and pathological conditions. Preliminary tensile tests carried out at higher strain levels showed tissue failure at about $11 \%$ strain. The elastic modulus can be evaluated at different strain levels for each subject and compared to assess possible differences in PF stiffness among samples from subjects with Ledderhose disease, diabetes and healthy subjects. In this work the secant modulus of different PF samples for each subject at $8 \%$ strain is calculated from experimental results of tensile tests and reported in Table 2. 


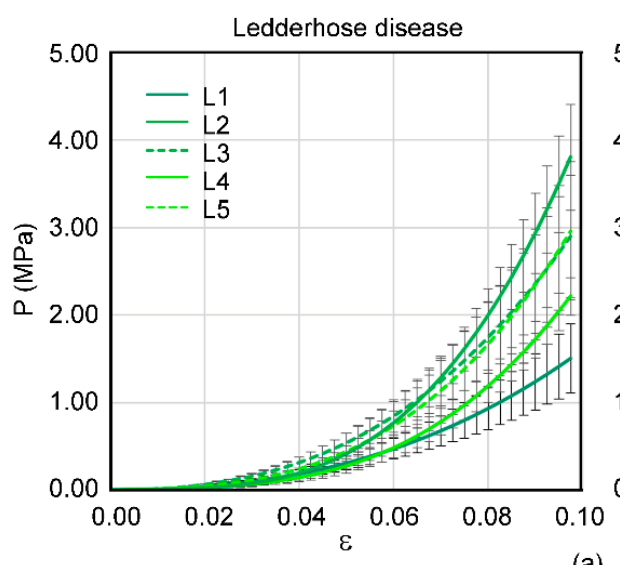

(a)

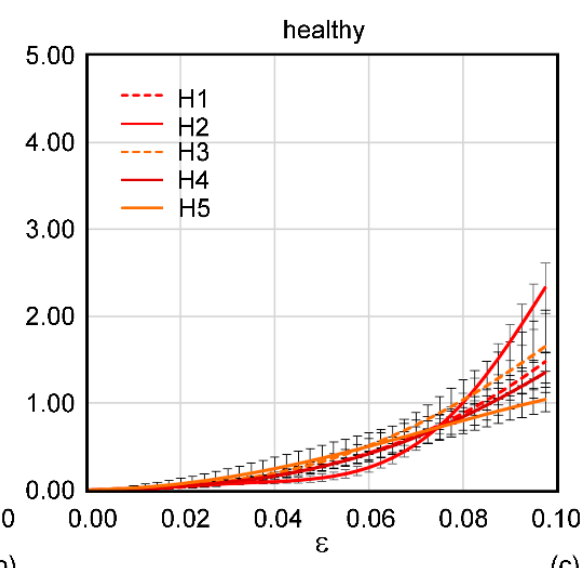

(b)

Figure 4. Results of tensile tests for PF of subjects with Ledderhose disease (a), diabetes (b) and healthy subjects (c). The results are reported in terms of nominal stress $P$ (mean values $\pm S E$ ) vs. nominal strain $\varepsilon$.

Table 2. Secant modulus $E_{S}$ of different PF samples for each subject at $8 \%$ strain from experimental results of tensile tests.

\begin{tabular}{ccccccccccccccc}
\hline \multicolumn{11}{c}{ ES $_{\mathbf{~}}(\mathbf{M P a})$} \\
\hline Sample & L1 & L2 & L3 & L4 & L5 & D1 & D2 & D3 & D4 & H1 & H2 & H3 & H4 & H5 \\
\hline$\# 1$ & 11.04 & 30.96 & 31.40 & 11.54 & 27.43 & 7.43 & 21.83 & 14.62 & 26.37 & 11.82 & 14.02 & 8.78 & 9.87 & 6.75 \\
$\# 2$ & 17.04 & 17.91 & 19.75 & 19.18 & 26.32 & 16.60 & 9.05 & 8.55 & 23.73 & 10.52 & 13.85 & 14.43 & 18.70 & 9.99 \\
$\# 3$ & 6.79 & 25.93 & 14.11 & 13.70 & 8.79 & 11.60 & 7.75 & 25.17 & 11.93 & 10.70 & 9.72 & 8.42 & 10.28 & 13.17 \\
\hline
\end{tabular}

Stress relaxation behavior is shown in Figure 5 for tests carried out at $8 \%$ strain, comparing PFs of patients with Ledderhose disease or diabetes and healthy subjects. The values of $P_{\text {norm }}$ at the end of the stress-relaxation test $(t=300 \mathrm{~s})$ show a stress reduction ranging from $30 \%$ to $60 \%$ of the initial stress value in subjects with Ledderhose disease, from $41 \%$ to $60 \%$ in subjects with diabetes and from $33 \%$ to $46 \%$ in healthy subjects.

The values of the secant modulus $\mathrm{E}_{\mathrm{S}}$ at $8 \%$ are compared to highlight possible differences among the three groups of involved subjects (Figure 6a). The ANOVA of the secant modulus with the Kruskal-Wallis test shows that there is a statistically significant difference among the three tested groups ( $p$-value $=0.049$ ). Subsequent multiple comparisons highlight that the elastic modulus of PF is significantly higher in subjects with Ledderhose disease with respect to the healthy group $(p$-value $=0.041)$. To investigate possible differences in the time-dependent mechanical behavior, normalized stress $P_{\text {norm }}$ at the end of the stress-relaxation test are compared in Figure 6b. The ANOVA with the Kruskal-Wallis test shows a statistically significant difference among different groups $(p$-value $=0.028)$. Subsequent multiple comparisons highlight that the relaxed normalized stress of PF is significantly lower in subjects with diabetes with respect to the healthy group $(p$-value $=0.021)$. 

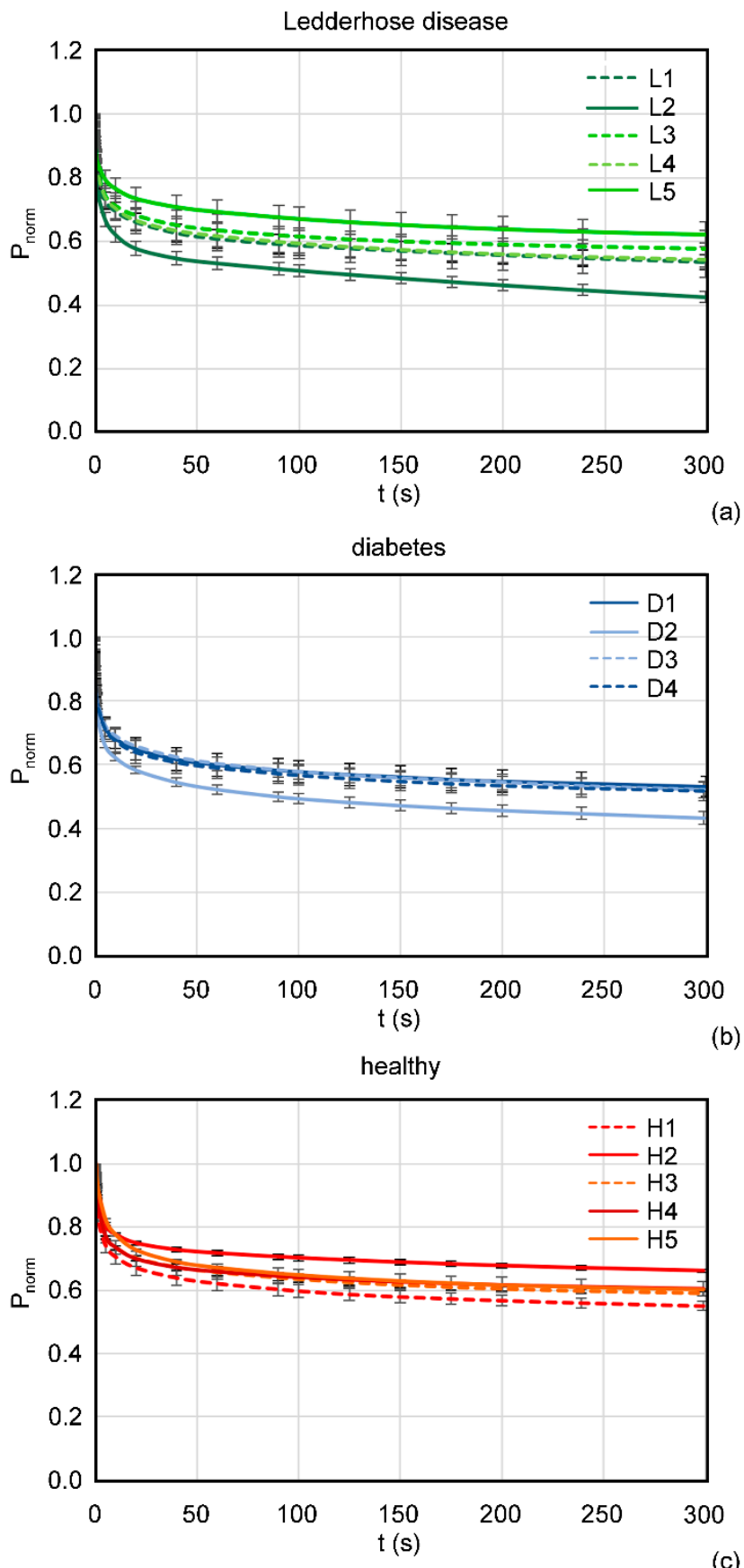

Figure 5. Results of stress relaxation tests at $8 \%$ strain, in terms of normalized stress $P_{\text {norm }}$ (mean values \pm SE) vs. time, for PF of subjects with Ledderhose disease (a), diabetes (b) and healthy subjects (c).

To consider possible aging-related differences in the mechanical properties, the age of the subjects is compared among the three groups (Figure 6c), highlighting a significant difference among them $(p$-value $=0.011)$.

Subsequent multiple comparisons reveal that subjects with Ledderhose disease involved in this study are significantly younger than the healthy ones ( $p$-value $=0.009)$. The correlation between $E_{S}$ and the Body Mass Index (BMI) shows a very low value of $\mathrm{R}^{2}$ (Figure $6 \mathrm{~d}$ ) and is not statistically significant ( $p$-value $=0.159$ ). An almost null value of $\mathrm{R}^{2}$ is found in the correlation between the secant elastic modulus and the thickness of the PF (Figure 6e). Moreover, a possible correlation between $E_{S}$ and age is evaluated, to 
understand if the age differences among groups of involved subjects could affect the results of this study. As shown in Figure 6f, a very low value of $R^{2}$ is found also in this case.

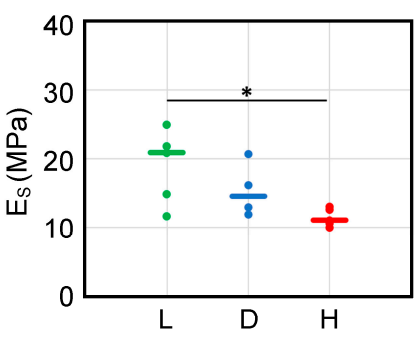

(a)

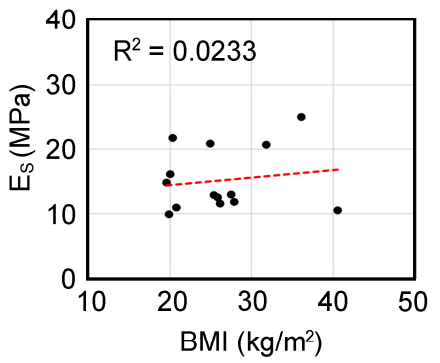

(d)

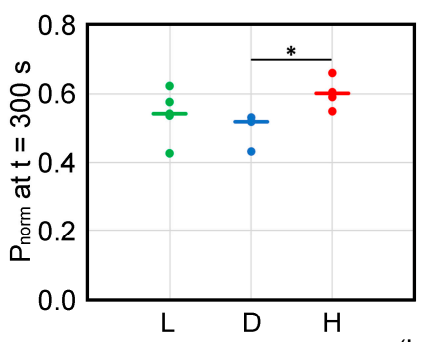

(b)

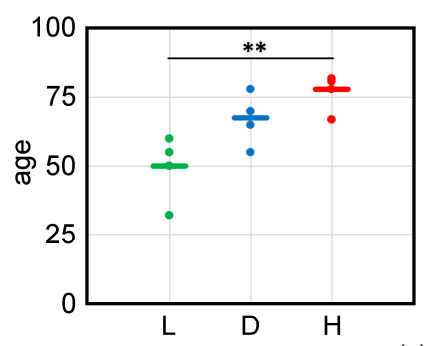

(c)
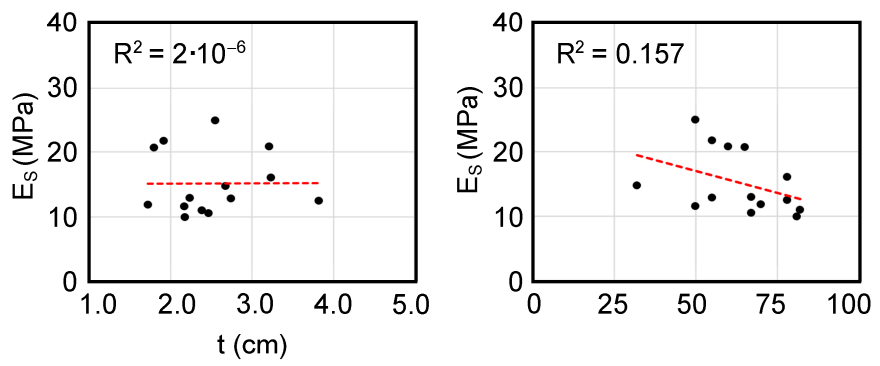

(e)

Figure 6. Comparison among PF samples from subjects with Ledderhose disease (L), diabetes (D) and healthy subjects $(\mathrm{H})$ : comparison of secant moduli $\mathrm{E}_{\mathrm{S}}(\mathbf{a})$; comparison of normalized stress value $\mathrm{P}_{\text {norm }}$ after $300 \mathrm{~s}$ of stress relaxation (b); comparison of age (c); correlation between secant modulus Es and BMI (d); correlation between secant modulus $\mathrm{E}_{\mathrm{S}}$ and $\mathrm{PF}$ thickness $\mathrm{t}(\mathbf{e})$; correlation between secant modulus $\mathrm{E}_{\mathrm{S}}$ and age (f). Differences among the data are indicated as statistically significant with $p$-value $<0.05\left(^{*}\right)$ or $p$-value $<0.01\left(^{* *}\right)$.

\section{Discussion}

In this study, the mechanical response of PF is investigated by experimental tests on the nonlinear stress-strain and time-dependent behavior, to understand if the Ledderhose disease and diabetes can affect PF biomechanics.

Concerning the nonlinear stress-strain response, the PF is stiffer in patients affected by Ledderhose disease and diabetes, with respect to healthy subjects. Indeed, secant elastic moduli $E_{S}$ reach considerable values in some PF samples (up to about $30 \mathrm{MPa}$ in L2 and about $26 \mathrm{MPa}$ in D4, see Table 2), while they are limited in the range between 10 and 14 MPa in healthy subjects. The same comparison can be gathered from Figure $6 \mathrm{a}$, where the values of mean $E_{S}$ are reported for each subject. This difference is statistically significant, as highlighted by ANOVA, with a source in the difference in the comparison of subjects affected by Ledderhose disease with healthy ones.

Previous studies hypothesized a possible correlation of PF stiffness with BMI [26] and PF thickness [21], which is here analyzed in Figure 6c,d. In these charts, the mean values for all the subjects are plotted together, irrespectively of the pathology, since data from the three groups are randomly positioned and not well grouped in distinct regions of the plot. Based on the experimental data of the present work, it appears that patients with greater BMI have tissue with slightly higher stiffness than patients with lower BMI, even if a good correlation is not found. Regarding the correlation of PF stiffness with thickness, this is absent in the present study.

Since the subjects included in the healthy group are significantly elderly with respect to subjects affected by Ledderhose disease and aging is generally considered responsible for variation in tissue mechanical properties [33], a possible correlation of PF stiffness with age is investigated. However, no correlation is found in the present work and this allows one to deduce that significant stiffness differences are mostly related to the pathology and not to the age of the subjects. 
Concerning the time-dependent behavior, the PF of healthy subjects shows a lower stress reduction than the PF taken from patients with Ledderhose syndrome or affected by diabetes. A statistically significant difference is found between diabetic and healthy subjects. In the literature there are very limited data on the viscoelastic properties of plantar aponeurosis for the comparison of the findings of this work with healthy patients. In a previous work [34], stress-relaxation phenomena were evaluated on plantar aponeurosis tissue from donors with age ranging from 67 to 78 years and not reporting specific foot pathologies. At $240 \mathrm{~s}$ of relaxation time, an average decrease of $40 \%$ (confidence interval: $38 \% \div 42 \%$ ) was estimated. This value is in line with that obtained in the present work, where a mean stress reduction of $44 \%$ and $33 \%$ of the initial stress value was measured at $240 \mathrm{~s}$, respectively, in $\mathrm{H} 1$ and H2, representing the samples with higher and lower stress reduction in the healthy subjects' group.

The findings of this study have to be seen in the light of some limitations. First, the number of involved subjects is limited. This limit is also probably the reason for the lack of statistically significance difference between diabetic and healthy groups, in spite of the fact that there is a tendency of diabetic PF to be stiffer than healthy PF (Figure 6a). Actually, the collection of PF from subjects who underwent excision of their intact plantar fascia from the foot during elective orthopedic surgery is not usual and it should be acknowledged that the present study represents the first attempt to get an insight into the differences in mechanical properties of PF depending on foot pathologies. The age differences among the groups of involved subjects were found because the healthy feet were dissected mainly from cadaveric subjects. The involvement of subjects of significantly different age among different groups is another limitation of this study, even though no correlation was found between PF stiffness and age in the present investigation. This aspect does not seem to be a bias in the experimental design.

In the present work, the maximum strength of the PF tissue was not considered, focusing on the viscoelastic properties in a physiologic range of tensile strain, which is below $10 \%$, according to in vivo experimental analyses [35]. An intrinsic limit of in vitro tests is represented by the impossibility of evaluating the effect of the basal tension on the mechanical response of plantar aponeurosis. As pointed out by tensile testing, the plantar tissue shows distinct nonlinear behavior, with an initial toe region followed by a stiffening. Consequently, a different value of the basal tension could result in a different response of the plantar tissue subjected to the same increment of tensile stress. This lack of information could be overcome by evaluating the basal tension of the plantar aponeurosis through in vivo elastosonography.

Due to the technique followed during dissection, it was not possible to evaluate the size of the whole PF of each patient. Therefore, there is a lack of information about the effective structural stiffness of $\mathrm{PF}$, since the mechanical tests made it possible to point out only local mechanical properties of the tissue. It can be recognized that PF acts mainly as a structure subjected to tensile forces in supporting the longitudinal foot arch [36]. Consequently, a transverse section and length of the plantar aponeurosis can have large effects on its structural behavior. This aspect can also affect the stress distribution within the foot under loading. To increase the knowledge about the above aspect it could be of interest to couple the proposed experimental testing with Magnetic Resonance (MR) acquisition to obtain information about the morphometric characteristics of the plantar aponeurosis.

\section{Conclusions}

In the present work the results of experimental tests on the nonlinear stress-strain and time-dependent behavior of PF show a tendency of plantar tissue in patients affected by Ledderhose syndrome and diabetes to be stiffer than the one in healthy subjects and to develop more intense stress-relaxation phenomena. The reduced number of samples considered in the present investigation comes from the evident difficulty in obtaining the PF from subjects that undergo foot excision due to Ledderhose disease or diabetes. This is confirmed also by the lack of similar data in the literature, up to now. 
The present work may be the first step to highlighting the importance of a deeper knowledge on the PF mechanical behavior both in healthy and pathological conditions. Moreover, the outlined experimental test method could be adopted in a multicentric study, thus allowing a large increase in the number of involved subjects.

The results of this study also represent an interesting basis for defining constitutive models able to describe the mechanical response of PF tissue and identifying the related constitutive parameters. Moreover, the development of in silico models of the foot with different PF mechanical behavior depending on the pathology will enable an analysis of possible alterations in foot biomechanics due to abnormal stiffening of PF tissue.

Author Contributions: Conceptualization, C.B. and P.G.P.; Methodology, P.G.P.; Software, S.T. and P.G.P.; Validation, S.T. and P.G.P.; Formal Analysis, S.T. and P.G.P.; Investigation, S.T., C.B. and P.G.P.; Resources, C.B. and P.G.P.; Data Curation, S.T. and P.G.P.; Writing-Original Draft Preparation, S.T.; Writing-Review \& Editing, P.G.P. and C.B.; Visualization, S.T. and P.G.P.; Supervision, P.R. and P.G.P.; Project Administration, P.R. and P.G.P. All authors have read and agreed to the published version of the manuscript.

Funding: This research received no external funding.

Institutional Review Board Statement: The study was conducted according to the guidelines of the Declaration of Helsinki, and approved by the Ethics Committee of the University of Padova and the National Institutes of Health (protocol code 3513/AO/15; date of approval: 28 January 2016).

Informed Consent Statement: Informed consent was obtained from all subjects involved in the study. Written informed consent has been obtained from the patients to publish this paper.

Data Availability Statement: The data presented in this study are available on request from the corresponding author. The data are not publicly available due to privacy and ethical restrictions.

Conflicts of Interest: The authors declare no conflict of interest.

\section{References}

1. Stecco, C.; Fede, C.; Macchi, V.; Porzionato, A.; Petrelli, L.; Biz, C.; Stern, R.; De Caro, R. The fasciacytes: A new cell devoted to fascial gliding regulation. Clin. Anat. 2018, 31, 667-676. [CrossRef]

2. Stecco, C.; Corradin, M.; Macchi, V.; Morra, A.; Porzionato, A.; Biz, C.; De Caro, R. Plantar fascia anatomy and its relationship with Achilles tendon and paratendon. J. Anat. 2013, 223, 665-676. [CrossRef]

3. Zwirner, J.; Zhang, M.; Ondruschka, B.; Akita, K.; Hammer, N. An ossifying bridge-On the structural continuity between the Achilles tendon and the plantar fascia. Sci. Rep. 2020, 10, 14523. [CrossRef]

4. Bolgla, L.A.; Malone, T.R. Plantar fasciitis and the windlass mechanism: A biomechanical link to clinical practice. J. Athl. Train. 2004, 39, 77-82.

5. Cheung, J.T.-M.; Zhang, M.; An, K.-N. Effects of plantar fascia stiffness on the biomechanical responses of the ankle-foot complex. Clin. Biomech. 2004, 19, 839-846. [CrossRef] [PubMed]

6. Monk, A.; Van Oldenrijk, J.; Riley, N.D.; Gill, R.H.; Murray, D. Biomechanics of the lower limb. Surgery 2016, 34, 427-435. [CrossRef]

7. Natali, A.N.; Pavan, P.G.; Stecco, C. A constitutive model for the mechanical characterization of the plantar fascia. Connect. Tissue Res. 2010, 51, 337-346. [CrossRef]

8. Pavan, P.G.; Pachera, P.; Stecco, C.; Natali, A.N. Constitutive Modeling of Time-Dependent Response of Human Plantar Aponeurosis. Comput. Math. Methods Med. 2014, 2014, 530242. [CrossRef]

9. Lemont, H.; Ammirati, K.M.; Usen, N. Plantar fasciitis: A degenerative process (fasciosis) without inflammation. J. Am. Podiatr. Med. Assoc. 2003, 93, 234-237. [CrossRef] [PubMed]

10. Bartold, S.J. The plantar fascia as a source of pain-biomechanics, presentation and treatment. J. Bodyw. Mov. Ther. 2004, 8, 214-226. [CrossRef]

11. Muñoz, J.P.; González, M.A.G.; García, J.C.C.; Nova, A.M. Relationship of the foot posture with the most frequent injuries in athletes. A pilot study. Arch. Med. Deport. 2015, 32, 76-81.

12. García-Gil, M.F.; Biosca, V.L. Plantar fibromatosis or Ledderhose disease: Diagnosis with ultrasonography. Radiología 2020, 62, 415-416. [CrossRef] [PubMed]

13. Young, J.R.; Sternbach, S.; Willinger, M.; Hutchinson, I.D.; Rosenbaum, A.J. The etiology, evaluation, and management of plantar fibromatosis. Orthop. Res. Rev. 2018, 11, 1-7. [CrossRef]

14. Carroll, P.; Henshaw, R.M.; Garwood, C.; Raspovic, K.; Kumar, D. Plantar Fibromatosis: Pathophysiology, Surgical and Nonsurgical Therapies: An Evidence-Based Review. Foot Ankle Spéc. 2018, 11, 168-176. [CrossRef] 
15. Fuiano, M.; Mosca, M.; Caravelli, S.; Massimi, S.; Benedetti, M.G.; Di Caprio, F.; Mosca, S.; Zaffagnini, S. Current concepts about treatment options of plantar fibromatosis: A systematic review of the literature. Foot Ankle Surg. 2019, 25, 559-564. [CrossRef]

16. Macgilchrist, C.; Paul, L.; Ellis, B.M.; Howe, T.E.; Kennon, B.; Godwin, J. Lower-limb risk factors for falls in people with diabetes mellitus. Diabet. Med. 2010, 27, 162-168. [CrossRef] [PubMed]

17. Paola, L.D.; Carone, A.; Vasilache, L.; Pattavina, M. Overview on diabetic foot: A dangerous, but still orphan, disease. Eur. Heart J. Suppl. 2015, 17, A64-A68. [CrossRef]

18. Whiting, D.R.; Guariguata, L.; Weil, C.; Shaw, J. IDF Diabetes Atlas: Global estimates of the prevalence of diabetes for 2011 and 2030. Diabetes Res. Clin. Pr. 2011, 94, 311-321. [CrossRef]

19. Arts, M.L.J.; De Haart, M.; Waaijman, R.; Dahmen, R.; Berendsen, H.; Nollet, F.; Bus, S.A. Data-driven directions for effective footwear provision for the high-risk diabetic foot. Diabet. Med. 2015, 32, 790-797. [CrossRef]

20. Biz, C.; Gastaldo, S.; Dalmau-Pastor, M.; Corradin, M.; Volpin, A.; Ruggieri, P. Minimally Invasive Distal Metatarsal Diaphyseal Osteotomy (DMDO) for Chronic Plantar Diabetic Foot Ulcers. Foot Ankle Int. 2018, 39, 83-92. [CrossRef]

21. Giacomozzi, C.; D'Ambrogi, E.; Uccioli, L.; Macellari, V. Does the thickening of Achilles tendon and plantar fascia contribute to the alteration of diabetic foot loading? Clin. Biomech. 2005, 20, 532-539. [CrossRef] [PubMed]

22. Guo, J.; Liu, X.; Ding, X.; Wang, L.; Fan, Y. Biomechanical and mechanical behavior of the plantar fascia in macro and micro structures. J. Biomech. 2018, 76, 160-166. [CrossRef] [PubMed]

23. Fausto de Souza, D.; Micaelo, L.; Cuzzi, T.; Ramos-e-Silva, M. Ledderhose disease: An unusual presentation. J. Clin. Aesthet. Dermatol. 2010, 3, 45-47. [PubMed]

24. Neagu, T.P.; Ţigliş, M.; Popescu, A.; Enache, V.; Popescu, Ş.A.; Lascăr, I. Clinical, histological and therapeutic modern approach of Ledderhose disease. Rom. J. Morphol. Embryol. 2018, 59, 691-697.

25. Fabrikant, J.M.; Park, T.S. Plantar fasciitis (fasciosis) treatment outcome study: Plantar fascia thickness measured by ultrasound and correlated with patient self-reported improvement. Foot 2011, 21, 79-83. [CrossRef]

26. Huerta, J.P.; García, J.M.A. Effect of gender, age and anthropometric variables on plantar fascia thickness at different locations in asymptomatic subjects. Eur. J. Radiol. 2007, 62, 449-453. [CrossRef]

27. Taş, S. Effect of Gender on Mechanical Properties of the Plantar Fascia and Heel Fat Pad. Foot Ankle Spéc. 2018, 11, 403-409. [CrossRef]

28. World Medical Association. World medical association declaration of Helsinki: Ethical principles for medical research involving human subjects. JAMA 2013, 310, 2191-2194. [CrossRef]

29. Schneider, C.A.; Rasband, W.S.; Eliceiri, K.W. NIH Image to ImageJ: 25 years of image analysis. Nat. Methods 2012, 9, 671-675. [CrossRef]

30. Carniel, E.L.; Albanese, A.; Fontanella, C.G.; Pavan, P.G.; Prevedello, L.; Salmaso, C.; Todros, S.; Toniolo, I.; Foletto, M. Biomechanics of stomach tissues and structure in patients with obesity. J. Mech. Behav. Biomed. Mater. 2020, 110, 103883. [CrossRef]

31. Jiang, M.; Lawson, Z.T.; Erel, V.; Pervere, S.; Nan, T.; Robbins, A.B.; Feed, A.D.; Moreno, M.R.; Veysel, E. Clamping soft biologic tissues for uniaxial tensile testing: A brief survey of current methods and development of a novel clamping mechanism. J. Mech. Behav. Biomed. Mater. 2020, 103, 103503. [CrossRef]

32. Todros, S.; Pianigiani, S.; De Cesare, N.; Pavan, P.G.; Natali, A.N. Marker Tracking for Local Strain Measurement in Mechanical Testing of Biomedical Materials. J. Med. Biol. Eng. 2018, 39, 764-772. [CrossRef]

33. Menz, H.B. Biomechanics of the Ageing Foot and Ankle: A Mini-Review. Gerontology 2014, 61, 381-388. [CrossRef]

34. Pavan, P.G.; Stecco, C.; Darwish, S.; Natali, A.N.; De Caro, R. Investigation of the mechanical properties of the plantar aponeurosis. Sur. Radiol. Anat. 2011, 33, 905-911. [CrossRef]

35. Gefen, A. The in vivo elastic properties of the plantar fascia during the contact phase of walking. Foot Ankle Int. 2003, 24, 238-244. [CrossRef] [PubMed]

36. Caravaggi, P.; Pataky, T.; Günther, M.; Savage, R.; Crompton, R. Dynamics of longitudinal arch support in relation to walking speed: Contribution of the plantar aponeurosis. J. Anat. 2010, 217, 254-261. [CrossRef] [PubMed] 